\title{
Mesoscopic Field and Current Compensator Based on a Hybrid Superconductor-Ferromagnet Structure
}

\author{
M. V. Milošević, G. R. Berdiyorov, and F. M. Peeters* \\ Departement Fysica, Universiteit Antwerpen, Groenenborgerlaan 171, B-2020 Antwerpen, Belgium
}

(Received 3 June 2005; published 30 September 2005)

\begin{abstract}
A rather general enhancement of superconductivity is demonstrated in a hybrid structure consisting of a submicron superconducting (SC) sample combined with an in-plane ferromagnet (FM). The superconducting state resists much higher applied magnetic fields for both perpendicular polarities, as the applied field is screened by the FM. In addition, FM induces (in the perpendicular direction to its moment) two opposite currents in the SC plane, under and aside the magnet, respectively. Because of the compensation effects, superconductivity persists up to higher applied currents. With increasing current, the sample undergoes SC-"resistive"-normal state transitions through a mixture of vortex-antivortex and phase-slip phenomena.
\end{abstract}

DOI: 10.1103/PhysRevLett.95.147004

In recent years, the superconductor-ferromagnet (SCFM) hybrids have received a lot of attention as one of the rare systems where ferromagnetism and singlet superconductivity coexist (for a review, see [1]). These hybrid structures are looked upon as candidates for futuristic nanoelectronics, combining superconducting circuits with magnetic storage elements. As a better understanding is needed, the ongoing studies are mainly focused on fundamental properties of nanoscale SC-FM samples and the plethora of related phenomena.

For example, although ferromagnetism in general suppresses superconductivity, direct SC-FM coupling appears to be crucial for the $\pi$-phase state with the critical current inversion in SC-FM-SC junctions [2] and Josephson current enhancement in SC-FM tunnel structures with very thin FM layers [3]. On the other hand, the nontrivial interplay between magnetism and superconductivity can be achieved even if SC and FM are not electronically coupled, as they still interact through the emerging magnetic fields. In that respect, arrays of submicron magnetic particles are used for applying well-defined local magnetic fields in the underlying superconductor [4]. One of the first applications of these nanomagnets was to engineer the pinning force of superconducting films, such that the critical current $j_{c}$ as a function of the applied magnetic field is increased due to a collective locking of the flux lattice to the magnetic array $[4,5]$. Since then, because of the technological relevance, enhancement of critical parameters in SC-FM heterostructures is of vast theoretical and experimental interest. Genenko et al., predicted theoretically an increased edge barrier critical current in superconductors completely surfaced by magnetic material [6]. In that case, a demagnetized magnetic layer acts as a magnetic screen, effectively shielding the Meissner state. Two years ago, Lange et al. measured a higher critical field in SC films regularly structured by out-of-plane magnetized dots [7]. However, this behavior strongly depended on the polarity of the applied field $\mathbf{H}_{\text {ext }}$ : for given FM-magnetization $\mathbf{M}$, an en-
PACS numbers: 74.78.Na, 74.25.Dw, 74.25.Op, 74.25.Sv

hancement of the critical parallel field $\left(\mathbf{H}_{\text {ext }} \| \mathbf{M}\right)$ was achieved at the expense of the antiparallel one. The same behavior was found both experimentally [8] and theoretically [9] in mesoscopic SC disks with an out-of-plane FM dot on top.

The first objective of the present Letter is to design a SC-FM hybrid structure where most critical properties can be tailored practically at will. For that matter, we consider a thin submicron superconducting sample with a ferromagnetic dot with in-plane magnetization on top (see Fig. 1). Such a device realization offers full exploitation of the magnetic flux pinning [10], dynamical properties of mesoscopic superconductors [11], and related vortex-antivortex phenomena [12]. Because of the opposite magnetic field at the poles of the magnet, the field-compensation effects lead to the critical field enhancement for both the positive and negative applied field (see Fig. 1). At the same time, our SC-FM sample might act as a current compensator as well: as a novel concept, the applied current is met by opposing FM-induced supercurrents, resulting in a larger critical current.

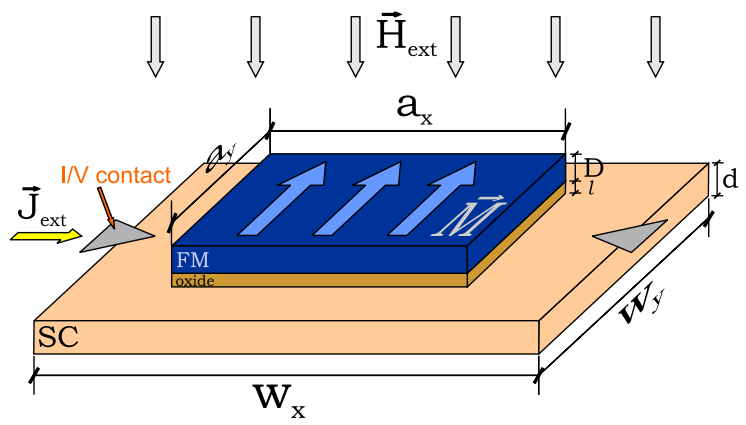

FIG. 1 (color online). A SC sample underneath a square FM dot with in-plane magnetization $\mathbf{M}$ (separated by an insulating layer). Depicted directions of $\mathbf{M}$, the applied external magnetic field $\mathbf{H}_{\text {ext }}$, and current $\mathbf{j}_{\text {ext }}$ are denoted as positive throughout the article. 
In our theoretical treatment of this system, we rely upon the Ginzburg-Landau (GL) formalism. In the stationary case we solve self-consistently two GL equations, derived from the Gibbs energy functional. For all details of this approach, we refer to Refs. [12,13].

To understand the dynamical properties of the device, we studied the current-voltage characteristics using the timedependent Ginzburg-Landau (TDGL) equation [14]

$$
\frac{u}{\sqrt{1+\Gamma^{2}|\Psi|^{2}}}\left(\frac{\partial}{\partial t}+\mathrm{i} \varphi+\frac{\Gamma^{2}}{2} \frac{\partial|\Psi|^{2}}{\partial t}\right) \Psi=(\nabla-\mathrm{iA})^{2} \Psi+\left(1-T-|\Psi|^{2}\right) \Psi,
$$

coupled with the equation for the electrostatic potential $\Delta \varphi=\operatorname{div}\left(\operatorname{Im}\left[\Psi^{*}(\nabla-\mathrm{iA}) \Psi\right]\right)$. Here, the distance is measured in units of the coherence length $\xi(0), \Psi$ is scaled by its value in the absence of magnetic field $\psi_{0}$, time by $\tau_{\mathrm{GL}}(0)=\pi \hbar / 8 k_{B} T_{c} u$, vector potential $\mathbf{A}$ by $c h / 2 e \xi(0)$, and the electrostatic potential by $\hbar / 2 e \tau_{\mathrm{GL}}(0) . \Gamma=$ $2 \tau_{E} \psi_{0} / \hbar$, with $\tau_{E}$ being the inelastic electron-collision time. For $\mathrm{Al}$ samples $\tau_{E} \sim 10 \mathrm{~ns}$, which results in $\Gamma \approx$ 1000. Parameter $u=5.79$ is taken from Ref. [14]. Note that in Eq. (1) the screening of the magnetic field is neglected, as we restrict ourselves to thin SC samples $(d<$ $\xi)$. The points where external current $j_{\text {ext }}$ is injected in the sample (see Fig. 1) were simulated as normal-metalsuperconductor contacts, i.e., with $\Psi=0$ and $-\nabla \varphi=$ $j_{\text {ext }}$. At the remainder of the sample edges, the Neumann boundary condition was used $\left(j_{\perp}=0\right)$.

We consider a square $\mathrm{Al}$ sample with parameters easily achievable with modern lithographic techniques: size $a_{x}=$ $a_{y}=1.5 \mu \mathrm{m}$, thickness $d=80 \mathrm{~nm}$, separated by an oxide layer of thickness $l=20 \mathrm{~nm}$ from the square FM with size $w_{x}=w_{y}=800 \mathrm{~nm}$ and thickness $D=50 \mathrm{~nm}$. The SC material is characterized by its coherence length at zero temperature, which we take as $\xi(0)=100 \mathrm{~nm}$ (typical value for mesoscopic Al samples [8]), and FM material by its saturation magnetization $M$. In our calculations, the FM is positioned at the center of the SC square, and since its stray field has opposite polarity at the FM-poles [see inset (a) in Fig. 2], the total flux $\Phi_{\mathrm{FM}}$ penetrating the SC equals zero. This feature inevitably leads to the appearance of vortex-antivortex configurations for sufficiently strong magnetization $M$ [12].

Figure 2 shows the Gibbs free energy [13] of the superconducting state, obtained after sweeping up/down the FM magnetization, where the number of induced vortexantivortex (VAV) pairs is denoted by Roman numbers. Note that these VAV states are the first found vortex states with zero total vorticity in finite mesoscopic SC samples. Insets (b),(c) in Fig. 2 show the $|\psi|^{2}$-density plots of successive VAV states. As vortices and antivortices are confined at the FM poles (where the stray field is maximal), they are effectively kept apart by the FM. In other words, the superconducting region under the FM always remains (anti)vortex-free. As a result, superconductivity can be sustained in the sample up to very large FM magnetization (as the slope $\partial \mathcal{G} / \partial M$ decreases in Fig. 2). Note that this is not the case if the FM has perpendicular magnetization, when total flux $\Phi_{\mathrm{FM}}$ captured by $\mathrm{SC}$ is positive and FM-induced vortices destroy superconductivity in the heart of the sample [8,9]. The experiment of Ref. [8] revealed that when such a sample is exposed to homogeneous external field $H_{\text {ext }}$, the $H_{\text {ext }}-T$ boundary is shifted towards positive fields due to the compensation with $\Phi_{\mathrm{FM}}$, resulting in a higher positive critical field (and consequently a reduced negative one).

In Fig. 3, the $H_{\text {ext }}-T$ superconducting/normal (S/N) phase boundary of our sample is shown, in the case of FM with bulk Co magnetization $M=1400 \mathrm{G}$ (solid line), compared to the case without FM (dashed line). The S/N boundary exhibits three novel features: (i) the $\mathcal{M}$-shaped boundary-the critical temperature for $H_{\mathrm{ext}}=0$ is reduced $\left(T_{\mathrm{cm} 0}\right)$, and is maximal for two symmetric nonzero $H_{\text {ext }}$ values $\left(T_{\mathrm{cm}}\right)$; (ii) for $T_{\mathrm{cm} 0}<T<T_{\mathrm{cm}}$ the N-S-N-S-N multireentrant behavior is observed during the $H_{\text {ext }}$ sweep; and (iii) substantial critical field enhancement is found for both $H_{\text {ext }}$ polarities.

The physical reason for these phenomena lies in the magnetic field compensation. In this particular case, for $H_{\mathrm{ext}}=0$, the $\mathrm{S} / \mathrm{N}$ transition at $T=T_{\mathrm{cm} 0}$ occurs for 2 vortex-antivortex pairs induced by the FM (state II in Fig. 2). Although their centers are pinpointed at the FM poles, these (anti)vortices are covering the whole sample as

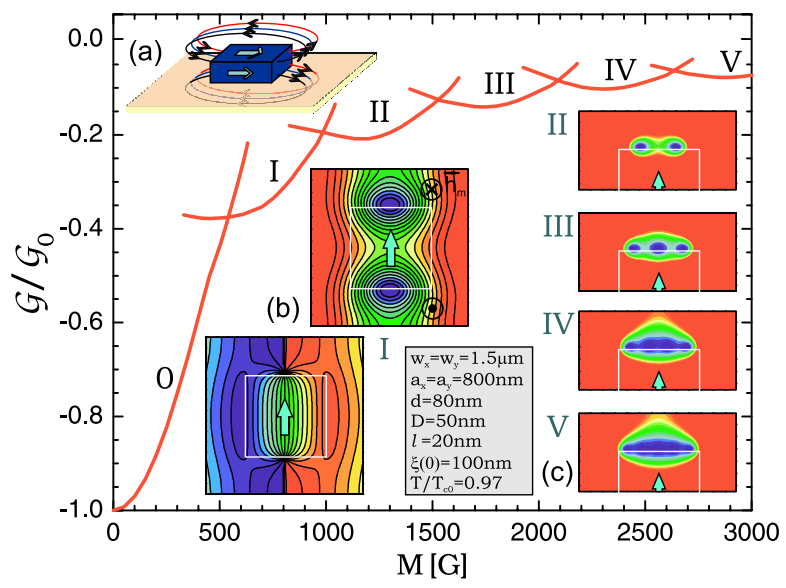

FIG. 2 (color online). The Gibbs free energy diagram $\left(G_{0}=\right.$ $\left.H_{c}^{2} V / 8 \pi\right)$ as a function of FM magnetization. Roman numbers denote the number of FM-induced vortex-antivortex pairs. Inset (a) illustrates the FM-stray field lines; (b) the Cooper-pair density [upper figure, darkest color-zero density (online blue/ red-low/high density)] and superconducting phase contour plot [gradation of gray color shows the circulation of phase $0-2 \pi$ (online blue/red $-0 / 2 \pi$ phase)] for state I; (c) the $|\psi|^{2}$-density plots (scale adjusted for clarity) under the positive pole of the magnet, for states II to V (FM edge depicted by white lines). 
$\xi(T)$ becomes large. When $H_{\text {ext }}>0$ is applied, the external flux is "absorbed" by the FM-induced antivortex. This effectively recovers superconductivity, and increases $T_{c}$. Each kink in the Little-Parks-like S/N boundary with increasing $H_{\text {ext }}$ corresponds to a change in total vorticity of $\Delta L=1$, where external flux lines are first annihilated by FM-induced antivortices; and in the absence of antivortices pinned on the positive pole of the FM, where the stray field and $H_{\text {ext }}$ are aligned (as found in Refs. [10]). In the latter case, each additional vortex suppresses superconductivity and $T_{c}$ decreases. However, the SC state remains protected at the opposite pole of the magnet which results in significantly higher critical field. In Fig. 3, an enhancement as high as $\sim 40 \%$ is achieved. This percentage can be even larger, if stronger magnetic materials are used (note high $M_{\text {cr }}$ in Fig. 2). The reduced zero-field critical temperature $T_{\mathrm{cm} 0}$ is only within few percent from $T_{c 0}$, but maximal $T_{\mathrm{cm}}$ (at $H_{\text {ext }}=1.97 \mathrm{mT}$ ) is several percent higher than the corresponding $T_{c}$ value in the absence of the FM.

For $H_{\text {ext }}<0$, the scenario is completely analogous, and the $\mathrm{S} / \mathrm{N}$ boundary is therefore symmetric. Note that this symmetry directly leads to feature (ii), which is actually a very rare magnetic field-induced-superconductivity (FIS) phenomenon. Similar unconventional behavior was reported earlier for materials like (EuSn) $\mathrm{Mo}_{6} \mathrm{~S}_{8}$ and $\lambda$-(BETS $)_{2} \mathrm{FeCl}_{4}$, and for SC films with out-of-plane FM arrays on top [7]. However, our sample holds a unique property-FIS is achieved for both perpendicular polarizations of applied field $H_{\text {ext }}$ (e.g., Fig. 3 for $\left.T=0.985 T_{c 0}\right)$.

Obviously, the above described phenomena are directly related to the strongly inhomogeneous FM-stray field (with zero average). Yet another interesting feature can be found in the stray-field-induced currents $\mathbf{j}_{\mathrm{FM}}$. Because of the field

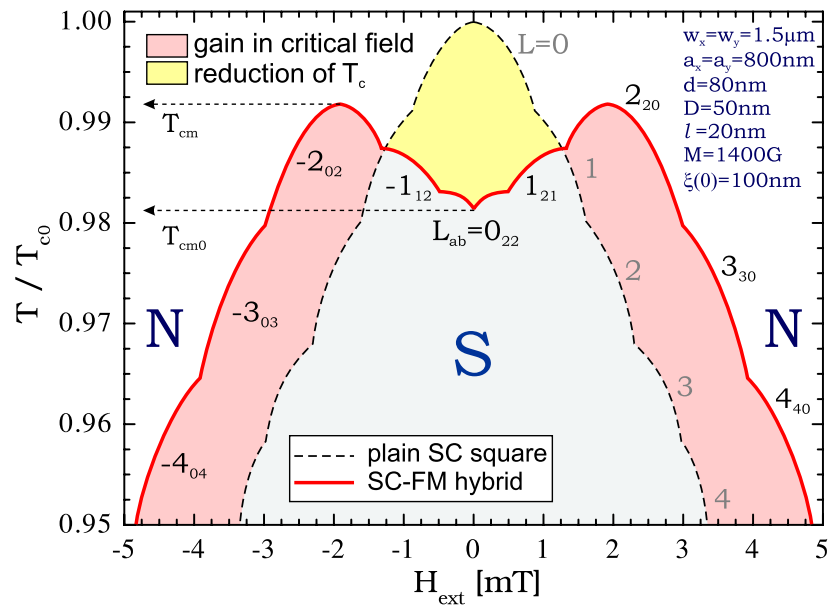

FIG. 3 (color online). The $\mathrm{S} / \mathrm{N}$ phase boundary for a square SC sample, without (dashed curve) and with (solid curve) an inplane FM dot on top. The total vorticity of the sample is denoted by $L$, while indices $a, b$ show the number of vortices and antivortices (induced and/or pinned by FM) at corresponding FM poles, respectively, $(L=a-b)$. landscape, these currents are actually circulating around the poles of the magnet, which ultimately results in two opposite current flows, under and aside the FM, in a direction perpendicular to the FM polarization [see Fig. 4(a)]. Obviously, with increasing FM magnetization, the amplitudes of $\mathbf{j}_{\mathrm{FM}}$ grow [as shown in Fig. 4(b)], until the depairing current is reached and the VAV pair nucleates at the FM poles. Consequently, $\mathbf{j}_{\mathrm{FM}}$ completely reverses [Fig. 4(b), $M=650 \mathrm{G}$ line], but changes polarity again with increasing $M$, before the appearance of the following VAV pair (see Fig. 2). This dual, steplike $\mathbf{j}_{\mathrm{FM}}$ profile may strongly affect the response of the device on the applied current in the $x$ direction (see Fig. 1). In order to investigate the critical current and dynamical properties of the system, we employ TDGL formalism. The key results are shown in Fig. 5, as differential resistance (obtained from calculated $I-V$ characteristics) as a function of applied current $j_{\text {ext }}$. Two critical currents, denoted as $j_{c 1}$ and $j_{c 2}$ in Fig. 5, can be identified. $j_{c 1}$ is the current at which the sample loses its zero resistance and transits to the so-called resistive state. $j_{c 2}$ has the more conventional meaning of the current at which the SC state becomes unstable.

When external current is applied to a plain SC square [Fig. 5(a)], it is nonuniformly distributed in the sample, with its maxima at the side edges (see cartoon in the inset). It is at these weak points where the vortex nucleates when the depairing current is reached (for corresponding $\left.j_{\text {ext }}=j_{c 1}\right)$. Because of the Lorentz force, this vortex is
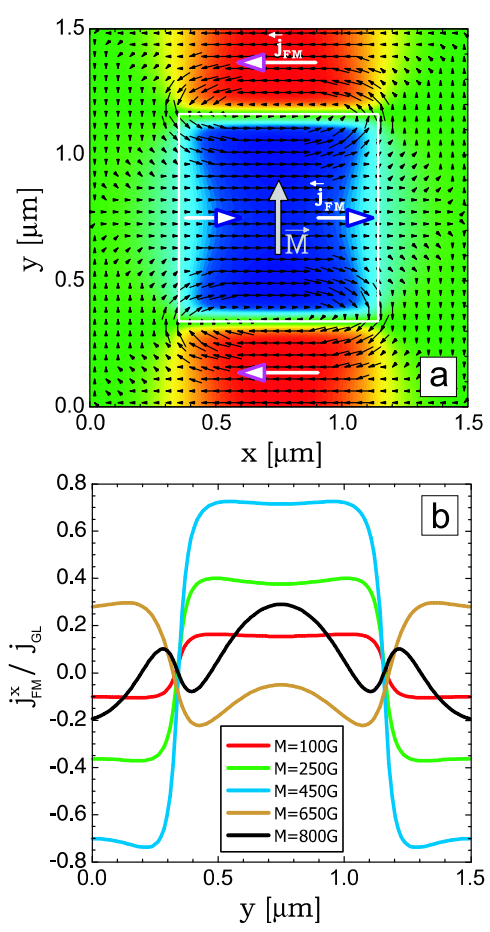

FIG. 4 (color online). (a) Vector plot of the SC-current $\mathbf{j}_{\mathrm{FM}}$ induced by an in-plane FM (Meissner state), superimposed on the contour plot of $j_{\mathrm{FM}}^{x}$ component. (b) The $j_{\mathrm{FM}}^{x}$ profile in the central cross section for different FM magnetization $\left(T=0.97 T_{c 0}\right)$. 

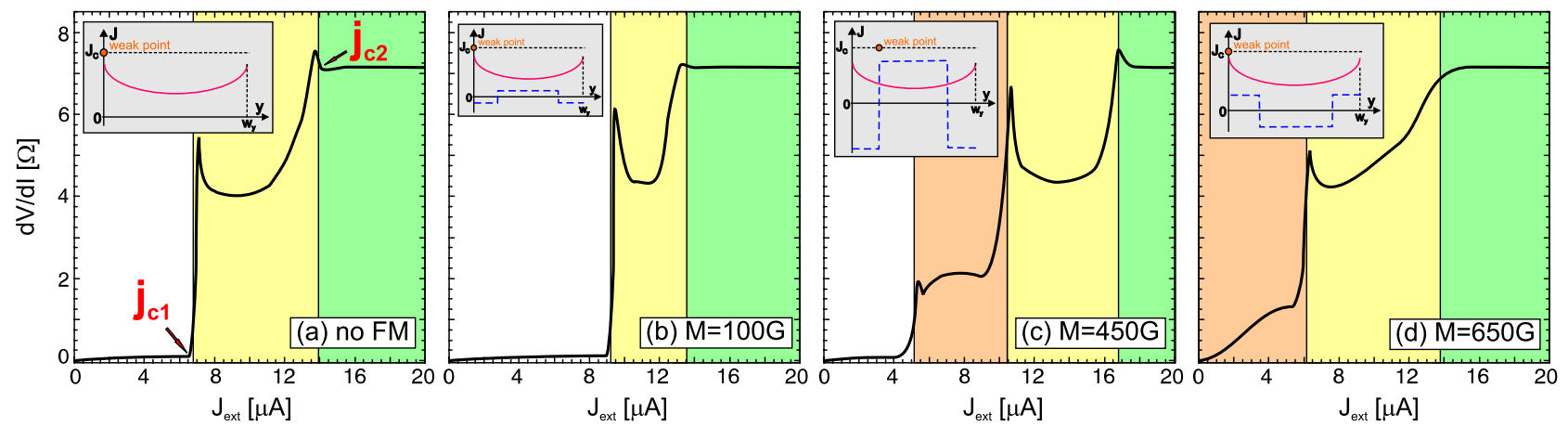

FIG. 5 (color online). Calculated differential resistance of the sample as function of the applied DC current, for different FM magnetization $\left(T=0.97 T_{c 0}\right)$. White areas denote the Meissner state, light gray (yellow) - resistive, medium gray (orange) — vortexantivortex, and dark gray (green)_normal state. Insets illustrate schematically the distribution of the applied (solid line) and FM-induced current ( $j_{\mathrm{FM}}^{x}$ component, dashed line) across the sample.

expelled across the sample, and subsequently nucleating again. To prevent the destruction of superconductivity, the SC phase exhibits a jump of $2 \pi$ at this "phase-slip line" [15]. This phase slip can be theoretically interpreted as an infinitely fast moving vortex. With continuous increase of $j_{\text {ext }}$, the current further suppresses the order parameter at the contacts (see Fig. 1), finally establishing a normal path between them (for $j_{\text {ext }}=j_{c 2}$ ) and normal-metal resistance is reached in Fig. 5(a). Note that the presence of $\mathrm{S} / \mathrm{N}$ contacts in our simulation gives small, but finite resistance even for $j_{\mathrm{ext}}<j_{c 1}$.

In the case of our SC-FM device, for small $M$, the FM induces current opposite to the external one, along the sample edges. Therefore, the resulting current at the weak points is decreased, and the critical current for vortex entry (phase-slip and non-zero resistance) increases. Figure 5(b) shows exquisite $j_{c 1}$ enhancement of $\sim 35 \%$, for $M=100 \mathrm{G}$ [see Fig. 4(b) for $\mathbf{j}_{\mathrm{FM}}$ profile]. However, for larger FM magnetization, after superposition of $j_{\mathrm{ext}}$ and $\mathbf{j}_{\mathrm{FM}}$, although edge current is further suppressed, the depairing current may be reached under the FM where $\mathbf{j}_{\mathrm{FM}}$ is maximal. As a result, the VAV pair nucleates, and SC transits to the resistive state for a low $j_{c 1}$ value [Fig. 5(c)]. Following VAV creation, $\mathbf{j}_{\mathrm{FM}}$ changes polarity and now compensates $j_{\mathrm{ext}}$ between the contacts instead of at the edges. Eventually, with a further increase of $j_{\mathrm{ext}}, \mathbf{j}_{\mathrm{FM}}$ is overwhelmed by the applied current, and the VAV pair is expelled from the sample; the influence of FM becomes negligible as further scenarios resemble the one of Fig. 5(a): current is again maximal at the edge, phase slip occurs, and superconductivity is destroyed. However, due to described VAV nucleation and current compensation between the contacts, for $M=450 \mathrm{G}$ we obtained a remarkable enhancement of $j_{c 2}$ of $\sim 21.5 \%$ [Fig. 5(c)].

For higher $M$, FM may induce a VAV pair in the sample [Fig. 2(b)]. In that case, even for very low applied current, a finite resistance was found $\left(j_{c 1}=0\right)$. This feature can serve as a tool for experimental detection of VAV pairs in contrast to the Meissner state in SC-FM hybrids. For certain value of $j_{\mathrm{ext}}$, the vortex and antivortex are depinned and leave the sample, followed by an immediate phase slip and consequent transition to the normal state. Because of the absence of the zero-resistance state, both $j_{c 1}$ and $j_{c 2}$ are significantly decreased [Fig. 5(d)].

In conclusion, we proposed a SC-FM device where both critical field and critical current can be substantially enhanced. Although our dynamical simulations are valid only in close vicinity of $T_{c}$, the main idea is generally applicable. Detailed influence of parameters and different dynamic regimes will be analyzed in a separate article.

This work was supported by the Flemish Science Foundation (FWO-Vl), the Belgian Science Policy, the JSPS/ESF-NES and the ESF-AQDJJ programs. Discussions with D. Y. Vodolazov are gratefully acknowledged.

*Electronic address: Francois.Peeters@ua.ac.be

[1] I. F. Lyuksyutov and V.L. Pokrovsky, Adv. Phys. 54, 67 (2005).

[2] V. V. Ryazanov et al., Phys. Rev. Lett. 86, 2427 (2001).

[3] F. S. Bergeret et al., Phys. Rev. Lett. 86, 3140 (2001).

[4] J. I. Martín et al., Phys. Rev. Lett. 79, 1929 (1997).

[5] D. J. Morgan and J. B. Ketterson, Phys. Rev. Lett. 80, 3614 (1998).

[6] Yu. A. Genenko et al., Phys. Rev. Lett. 83, 3045 (1999).

[7] M. Lange et al., Phys. Rev. Lett. 90, 197006 (2003).

[8] D. S. Golubović et al., Europhys. Lett. 65, 546 (2004).

[9] M. V. Milošević et al., Phys. Rev. B 66, 024515 (2002).

[10] M. J. Van Bael et al., Phys. Rev. Lett. 86, 155 (2001); M. V. Milošević et al., Phys. Rev. B 69, 104522 (2004).

[11] B. I. Ivlev and N. B. Kopnin, Adv. Phys. 33, 80 (1984).

[12] M. V. Milošević and F. M. Peeters, Phys. Rev. Lett. 93, 267006 (2004); Phys. Rev. Lett. 94, 227001 (2005).

[13] V. A. Schweigert et al., Phys. Rev. Lett. 81, 2783 (1998).

[14] L. Kramer and R. J. Watts-Tobin, Phys. Rev. Lett. 40, 1041 (1978).

[15] D. Y. Vodolazov et al., Phys. Rev. B 71, 184502 (2005). 